\title{
Las políticas educativas TIC en España después del Programa Escuela 2.0: las tendencias que emergen
}

\section{ICT education policies in Spain after School Program 2.0: Emerging Trends}

\author{
Manuel Area Moreira ${ }^{1}$, Cristina Alonso Cano ${ }^{2}$, José Miguel Correa Gorospe ${ }^{3}$, María Esther del \\ Moral Pérez ${ }^{4}$, Juan de Pablos Pons ${ }^{5}$, Joaquín Paredes Labra ${ }^{6}$, José Peirats Chacón ${ }^{7}$, Ana Luisa \\ Sanabria Mesa ${ }^{8}$, Angel San Martín Alonso ${ }^{9}$ y Jesús Valverde Berrocoso ${ }^{10}$ \\ ${ }^{1,8}$ Universidad de La Laguna, ${ }^{2}$ Universidad de Barcelona, ${ }^{3}$ Universidad del País Vasco, ${ }^{4}$ Universidad de Oviedo, ${ }^{5}$ Universidad de Sevilla, \\ ${ }^{6}$ Universidad Autónoma de Madrid, ${ }^{7,9}$ Universidad de Valencia y ${ }^{10}$ Universidad de Extremadura (España).
}

E-mail: manarea@ull.edu.es; cristina.alonso@ub.edu; jcorrea@sc.ehu.es; emoral@uniovi.es; jpablos@us.es; joaquin.paredes@uam.es; Jose.Peirats@uv.es; asanabri@ull.edu.es; Angel.Sanmartin@uv.es; jevabe@unex.es

\section{Información del \\ artículo \\ Recibido 11 de Noviembre de 2014. Revisado 9 de \\ Diciembre de 2014. \\ Aceptado 15 de Diciembre de 2014.}

\section{Palabras clave:}

Política educativa, Escuela 2.0, modelo 1:1, Tecnología Educativa, TIC

\section{Keywords:}

Eductional Policy, School 2.0, 1:1 model, Educational Technology, ICT

\section{Resumen}

En este artículo se analiza la situación actual de las políticas educativas destinadas a la incorporación de las TIC a las escuelas en una muestra de ocho comunidades autónomas de España (Andalucía, Canarias, Cataluña, Extremadura, Madrid, País Valenciano, País Vasco y Principado de Asturias) después del proceso impulsado por el Programa Escuela 2.0. Este programa estuvo vigente durante el periodo 2009-2012 y respondió al modelo 1:1, de un ordenador por estudiante. Este análisis se guió por las siguientes cuestiones: ¿Qué efectos permanecen de las experiencias de políticas educativas del modelo 1:1?; ¿Qué nuevos proyectos o programas están vigentes en los sistemas educativos de las comunidades autónomas? ¿Qué nuevas tendencias con relación a las TIC están emergiendo en la agenda educativa de las políticas autonómicas? Se concluye que las nuevas políticas TIC, entre otras acciones, apuntan a centrar su atención en la incorporación de las PDI a todas las aulas, a la dotación de tabletas a los estudiantes en vez de miniportátiles, a políticas de sustitución de los libros de texto de papel por plataformas de contenidos digitales y a la creación de portales de recursos en la nube. Se constata también la ausencia de un programa o política educativa TIC coordinada a nivel de todo el estado como fue la experiencia del Programa Escuela 2.0.

\section{A b stract}

In this article the current situation of educational policies for the incorporation of ICT in schools in a sample of eight regions of Spain (Andalusia, Canary Islands, Catalonia, Extremadura, Madrid, Valencia, the Basque Country and the Principality of analyzes Asturias) after the process driven by the Escuela 2.0 program. This program was in effect during the period 2009-2012 and responded to the 1:1 model of a computer per student. This analysis was guided by the following questions: What effects remain from the experiences of educational policies of the 1:1 model?; What new projects or programs are in place in the educational systems of the regions? What new trends in relation to ICT are emerging in the educational agenda of regional policies? We conclude that the new ICT policy, among other things, aim to focus on the integration of IDPs to all classrooms to equipping students tablets instead of netbooks, a substitution policies textbooks paper for digital content platforms, to create portals of cloud resources. The absence of an educational program or policy level ICT coordinated statewide as was the experience of School Program 2.0 has been observed. 


\section{Introducción}

Las políticas educativas destinadas a incorporar las TIC a las escuelas en el contexto español tienen una trayectoria histórica de más de un cuarto de siglo (Area, 2006). En una primera etapa en los años ochenta del siglo pasado, estas políticas fueron impulsadas por el gobierno central español. El programa de referencia de aquella época fue el denominado Atenea, posteriormente reconvertido en PNTIC (Programa Nacional de Tecnologías de la Información y Comunicación). A continuación, con la creación y consolidación de los gobiernos de las Comunidades Autónomas y la cesión de competencias en materia educativa, se elaboraron políticas y programas de ámbito regional, que se desarrollaron entre la última década del siglo XX y la primera del XXI. Durante dicho periodo las políticas educativas autonómicas para integrar las TIC en las escuelas se plantearon y ejecutaron sin que existieran objetivos o acciones compartidas entre unos gobiernos regionales y otros. Eran políticas que seguían directrices europeas, porque en parte venían financiadas desde la Unión Europea, pero aunque coincidían en muchas acciones (dotación de salas de informática a los centros, formación del profesorado, producción de materiales educativos digitales), se desarrollaron separadamente sin un plan o proyecto consensuado para todo el territorio español. Sin embargo, durante un breve periodo de tres años (2009-12), asistimos, en nuestro país, a una experiencia de política nacional coordinada. Bajo el paraguas de lo que se denominó el «Programa Escuela 2.0» ${ }^{1}$ se compartieran metas, procesos y presupuestos similares en la mayor parte de las comunidades autónomas de España. Fue una experiencia breve, interrumpida bruscamente, pero intensa en sus acciones y polémica en su debate.

\section{La política del modelo 1:1 en España. El Programa Escuela 2.0 (2009-2012)}

El contexto, surgimiento y propuesta del Programa Escuela 2.0 en nuestro país estuvo enmarcado en un contexto mundial que, a mediados de la primera década del siglo XXI, comenzó a desarrollar políticas destinadas a incorporar mucha tecnología a las aulas. Estas políticas empezaron a configurar lo que se conoció como el «modelo 1 a 1», es decir, un ordenador por niño (OCDE, 2010). Las mismas, inicialmente, surgieron en los países más desarrollados del planeta (América del Norte, Europa y países de la cuenca del Pacífico, como Japón y Corea del Sur). Paralelamente, impulsado por el MIT (Massachusetts Institute of Technology), se impulsó el proyecto de OLPC (One Laptop per Child, 2011) destinado a dar computadoras baratas a cada estudiante de los sistemas escolares de países en desarrollo ${ }^{2}$. El caso del «Plan Ceibal» en Uruguay, todavía vigente, es la experiencia internacional más conocida y probablemente más exitosa, ya que han logrado que cada alumno de todas las etapas y cursos educativos dispongan de su computadora portátil personal (Fullan, Watson y Anderson, 2013). Véase también una revisión de estas políticas en América Latina en Lugo (2012).

Por otra parte, desde la Unión Europea se propició y se financió que los distintos países integrantes de la misma invirtieran en la introducción de las tecnologías digitales a las escuelas. Estas políticas fueron impulsadas por instancias como European Schoolnet (EUN) que apoyaron decididamente el modelo 1:1. Una recopilación y análisis de los proyectos que bajo el modelo 1:1 se desarrollaron en países europeos puede verse en Balanska, Bannister, Hertz, Sigillò, \& Vuorikari (2013).

En pocas palabras, pudiéramos señalar que el programa Escuela 2.0 no fue una experiencia de política educativa TIC aislada de lo que estaba ocurriendo en el contexto mundial y europeo, sino todo lo

\footnotetext{
Vease el portal web del INTEF donde se resumen algunas de las características de dicho programa en http://www.ite.educacion.es/escuela-20

2 Vease el portal web de la organización OLPC en http://one.laptop.org/
} 
contrario. Representó una apuesta española destinada a facilitar, de forma masiva, el acceso a las TIC a todos los escolares y propiciar que el profesorado integrase pedagógicamente las mismas en su práctica de aula.

Como ya hemos señalado, esta política del modelo 1:1 tuvo una corta existencia temporal. Fue impulsada por el gobierno de España durante el periodo 2009-2012. Este Programa surgió como una iniciativa del denominado «Plan-E» destinado a la reactivación de la economía española. Tuvo un presupuesto inicial de 200 millones de euros, cofinanciados entre el gobierno central y las Comunidades Autónomas, y se destinó, en principio, a los alumnos de $5^{\circ}$ y $6^{\circ}$ de Educación Primaria, aunque en Cataluña y Extremadura se centró en los primeros cursos de la Educación Secundaria Obligatorio (ESO).

Otro dato relevante es que este modelo de política educativa de un ordenador por estudiante no fue adoptado por todas las comunidades autónomas españolas, ya que los gobiernos autónomos eran competentes para adoptarla o no. De este modo las Comunidades Autónomas que participaron en la implementación del programa Escuela 2.0 fueron Andalucía, Aragón, Asturias, Cantabria, Castilla-León, Castilla-La Mancha, Cataluña, Galicia, Extremadura, Islas Baleares, Islas Canarias, La Rioja, Navarra, Murcia, País Vasco, así como las ciudades autónomas de Ceuta y Melilla.

Las comunidades autónomas que no quisieron adoptar dicha política fueron las de Madrid y Valencia. En consecuencia, durante el periodo aludido (2009-2012) se dieron simultáneamente dos procesos diferenciados de implantación de políticas educativas destinadas a la incorporación de las TIC en las escuelas. Uno, en quince comunidades autónomas, que respondía al modelo 1:1 «Programa Escuela 2.0», y otro en dos comunidades autónomas que optaron por el modelo selectivo de «Centro Inteligente» o «Instituto de Innovación», que identifica a una serie de colegios-piloto o experimentales, a los que se dotaba de abundante tecnología en todas las aulas de dicho centro.

La llegada del Partido Popular al gobierno nacional a finales de 2011, junto con los importantes recortes presupuestarios para reducir el déficit público, supusieron la supresión del Programa Escuela 2.0 en el año 2012.

\section{La situación de las políticas educativas TIC después de finalizar el Programa Escuela 2.0 en una muestra de Comunidades Autónomas (2012-14)}

¿Qué efectos se observan en la actualidad como resultado del programa Escuela 2.0 y las otras políticas TIC en cada Comunidad Autónoma? ¿Cuál es la situación actual de las políticas educativas sobre tecnología educativa? ¿Cuáles son las acciones/programas que actualmente se están impulsando desde las Consejerías de Educación de las Comunidades Autónomas? ¿Cuáles son las nuevas tendencias de dichas políticas a corto y medio plazo?

Estas son las preguntas clave a las que intenta responder este artículo. Para ello hemos realizado una selección de aquellos casos de comunidades autónomas españolas sobre las que teníamos información contrastada los investigadores firmantes de este artículo ${ }^{3}$.

El análisis que presentamos se circunscribe a ocho casos: seis que participaron e implementaron el modelo 1:1, a través del denominado «Programa Escuela 2.0» (en concreto son Andalucía, Asturias, Cataluña, Canarias, Extremadura y País Vasco) y las otras dos que desarrollaron otra política TIC, es decir, Madrid y Valencia. Las seis primeras coinciden en que este Programa comenzó focalizando su

\footnotetext{
Los autores de este trabajo hemos desarrollado varios proyectos I+D financiados por el Plan Nacional de I+D que tuvieron como objeto de estudio la implantación de estas políticas de incorporación de las TIC en el sistema escolar en distintas comunidades autónomas. Algunas de sus publicaciones sobre este particular pueden consultarse en Alonso (2012); Alonso, Guitert y Romeu (2014); Area y Sanabria (2014); De Pablos (2012); De Pablos, Area, Correa y Valverde (2010); Losada, Karrera y Correa, J. M. (2011); Paredes (2012); Peirats, Sales y San Martín (2009): Sanabria y Area (2011); Valverde (2014).
} 
atención en la dotación de un ordenador por estudiante en los cursos de $5^{\circ}$ y $6^{\circ}$ de Educación Primaria, a excepción de Cataluña y Extremadura que destinaron los equipamientos a la Educación Secundaria Obligatoria. En cada comunidad el «Programa Escuela 2.0» adoptó señas de identidad propias en su denominación. De este modo, el «Programa Escuela 2.0» en Asturias se vinculó a «Educastur» ${ }^{4}$, en Andalucía se llamó «Escuela TIC2.0», en Canarias «clic-Escuela 2.0» ${ }^{5}$, en Cataluña recibió el nombre de «Educat 2.0» ${ }^{6}$, y en el País Vasco se denominó «Eskola 2.0» ${ }^{7}$. En líneas generales coinciden que sus objetivos de actuación se centraron en la dotación de ordenadores portátiles para el profesorado y alumnado, de PDI para las aulas, conectividad de los centros a Internet, creación y desarrollo de materiales educativos online y la formación del profesorado en TIC.

Por su parte, las otras dos comunidades no tenían un programa único y definido con relación a la incorporación de las TIC, sino programas y plataformas digitales que englobaban distintos servicios, proyectos, contenidos y herramientas educativas. En la Comunidad de Madrid se creó el sitio web «Educamadrid» ${ }^{8}$ que engloba numerosos servicios con relación a las TIC (informes, contenidos digitales, formación online, etc.). Dentro del mismo, se creó el programa denominado «Proyectos de Institutos de Innovación Tecnológica» que solamente abarcó a un número limitado de centros educativos previamente seleccionados. Por su parte en la Comunidad Autónoma de Valencia ha desarrollado varios programas educativos entre los que destacan el LluireX, destinado a implantar el sistema Linux en las escuelas de dicha comunidad, y sobre todo el denominado «Centro Educativo Inteligente» que fue la alternativa al «Programa Escuela 2.0». Este proyecto tuvo un limitado impacto y generalización en los colegios valencianos. Frente al modelo de un ordenador por alumno para todos en los cursos finales de la Educación Primaria, como era la meta del Escuela 2.0, el Programa «Centro Educativo Inteligente» se dirigió a una reducida selección de centros experimentales que recibieron una abundante dotación de tecnologías.

En este trabajo describiremos la situación actual, en estas ocho comunidades autónomas que hemos seleccionado y se responderán estas cuestiones:

a) ¿Qué efectos permanecen de las experiencias de políticas educativas del modelo 1:1?

b) ¿Qué nuevos proyectos o programas están vigentes en los sistemas educativos de las comunidades autónomas?

c) ¿Qué nuevas tendencias con relación a las TIC están emergiendo en la agenda educativa de las políticas autonómicas?

Metodológicamente este trabajo se desarrolló como un proyecto colectivo donde empleamos un espacio web compartido. Cada uno de los autores se responsabilizó de recoger los datos específicos de la política educativa de una comunidad autónoma guiados por las tres cuestiones anteriores y, a partir de los mismos, elaboró una síntesis de la situación en la que se encuentra. Posteriormente se redactaron las conclusiones intentando identificar las tendencias emergentes. A continuación se presenta la situación actual de las políticas educativas TIC después de la desaparición del Programa Escuela 2.0 en cada una de las comunidades autónomas que han sido analizadas.

\footnotetext{
4 http://blog.educastur.es/escuela20/

http://www.gobiernodecanarias.org/ceus/servicios/tecnologia_educativa_medusa/clic_escuela/

http://www.xtec.cat/web/innovacio/educat

http://www.eskola20.euskadi.net/

8 http://www.educa2.madrid.org/educamadrid/
} 


\subsection{Andalucía}

Andalucía cuenta con una importante trayectoria en el uso educativo de las TIC, que se ha mantenido en el tiempo. La introducción, hace diez años, de la política de centros TIC en Andalucía, ha tenido como efecto la «normalización» del profesorado, del alumnado y de las familias en el uso de las TIC; aunque el uso de las TIC no esté generalizado aún, ni mucho menos su utilización adecuada, desde un punto de vista pedagógico. Esa circunstancia ha dado lugar a que gran parte del profesorado entusiasta se agrupe en movimientos informales. Las redes sociales y las aplicaciones de microbloging (Twitter) han sido las vías de comunicación, información y convocatoria de todo tipo de actividades y eventos presenciales y virtuales. Dejar hacer a esos grupos, no tratar de capitalizar sus esfuerzos, pero poner todas las condiciones necesarias para que existan y se difundan sus experiencias, promover la experimentación y la innovación flexibilizando las exigencias burocráticas y permitiendo la formación entre iguales en horario laboral, serían elementos claves de una buena política decididamente a favor de la transformación de las prácticas educativas (Sola, 2014).

La llegada del «Programa Escuela 2.0», según se ha constatado, no ha terminado de revertir la situación, ni en cuanto a que la formación en TIC del docente signifique claramente un acicate para el aumento del uso de estas tecnologías en el aula, ni en cuanto a que su uso suponga en sí un paso adelante cualitativo, pues la formación impartida posee un carácter fundamentalmente técnico, sin aplicaciones didácticas. La formación, sigue muy centrada en la mera alfabetización digital, para quienes deben trascender el empleo de los medios informáticos a su dimensión pedagógica.

Respecto a cómo valoró el profesorado la política de implantación del programa Escuela TIC 2.0, realizada por la Comunidad Autónoma, cabe señalar que la dotación de ordenadores y demás recursos en las aulas es el aspecto más apreciado (el $43,6 \%$ de los profesores consultados así lo afirmaron). Otras vertientes importantes del Programa como la formación específica a los docentes, la difusión informativa, la creación de materiales o el apoyo institucional ofrecido por la Administración, se consideraron deficitarias (De Pablos, 2012).

Otro aspecto a señalar es que el paso al programa Escuela 2.0, supuso al mismo tiempo la universalización de las TIC en los centros andaluces y el abandono en la práctica de los "Centros TIC»; un programa que se había planteado la incorporación paulatina, a escuelas e institutos, de las TIC con al menos la asunción formal de proyectos educativos, y que fue sustituido por el reparto masivo de portátiles y, en opinión del profesorado, sin que se añadiera política alguna de carácter pedagógico.

Durante los cursos 2010-2011 y 2011-2012 el Servicio de Innovación Educativa puso en marcha con carácter experimental medidas encaminadas a la utilización de nuevos soportes de contenidos, recursos y herramientas TIC en el proceso de enseñanza aprendizaje. Para ello, se seleccionaron una serie de centros que bajo tres modalidades, on-line, off-line y elaboración de materiales propios, trabajaron con libros de textos y recursos en soporte digital. Esta experimentación comenzó en el curso 2010-2011 en $5^{\circ}$ y $6^{\circ}$ de Educación Primaria, y $1^{\circ}$ Educación Secundaria Obligatoria y continuó en 2011-2012 en las etapas $5^{\circ}$ y $6^{\circ}$ de Educación Primaria y $2^{\circ}$ de Educación Secundaria. Participaron un total de 98 centros públicos (82 centros por curso, distribuidos proporcionalmente por provincias y etapas). Los materiales de la modalidad online y offline fueron proporcionados por 22 editoriales (más de 200 títulos para las diferentes materias). Para la elaboración de materiales propios los centros contaban con la posibilidad de utilizar más de 200.000 ODE (Objetos Digitales Educativos) alojados en las plataformas Agrega y Averroes, así como el uso de plataformas educativas como Helvia o Moodle. 
Al inicio del curso escolar 2012/2013, la Consejería de Educación de la Junta de Andalucía planteó la realización de unas Jornadas para analizar lo que había supuesto la incorporación de las TIC en la mejora del proceso de enseñanza-aprendizaje, durante todo el período de funcionamiento de los Centros TIC y del Plan Escuela TIC 2.0 en Andalucía (VV.AA., 2012). Se buscaba detectar las fortalezas y debilidades de estos programas con objeto de obtener un documento de referencia que sirviese para orientar el futuro de las TIC en el sistema educativo andaluz. Para esta reflexión, la Consejería entendió que era necesario contar con todos los sectores de la comunidad educativa involucrados en los proyectos de integración de las TIC.

En todas las mesas de debate de las Jornadas se señalaron carencias y posibles líneas de actuación sobre la integración de las TIC en la práctica docente. Un aspecto a resaltar, entre otros, en las conclusiones ha sido la reivindicación de la figura del coordinador/a TIC, que debe recuperar sus funciones pedagógicas, ya que en estos diez años se ha convertido únicamente en la persona con un rol técnico en el centro, para el que además no está específicamente formado.

El gran desafío al que tienen que dar respuesta las políticas educativas de incorporación de las TIC a los centros educativos, no es únicamente el de dotarlos de herramientas informáticas, para que sean utilizadas por los estudiantes y el profesorado, sino que deben vincularse a un proyecto de reforma e innovación educativa general, lo que implica una remodelación del modelo formativo, de tal modo que contemplen al sistema educativo de manera integrada.

\subsection{Canarias}

Los antecedentes de las políticas educativas TIC en la comunidad Autónoma de Canarias se remontan a los años ochenta del siglo pasado con el «Proyecto Ábaco» y el «Programa de Medios Audiovisuales», le siguió el «Programa de Nuevas Tecnologías» en los años 90, y posteriormente, el «Programa Medusa» a finales de los 90 y principios del 2000. Desde entonces y hasta la actualidad, el denominado «Programa Medusa» asumió la función de gestionar y coordinar todas las iniciativas instituciones que se han desarrollado al amparo de los programas nacionales y europeos, como «Internet en la Escuela», «Internet en el Aula» y el recientemente desaparecido «Programa clic Escuela 2.0» que estuvo vigente hasta finales del año 2012.

La suspensión del programa nacional «Escuela 2.0» dejó sin apoyo y cobertura institucional el desarrollo del modelo 1:1 en todas las Comunidades Autónomas, pero no supuso la interrupción de la prácticas educativas para la integración de las tecnologías digitales en la educación escolar, ni en los contextos de centros ni de aula ni de la administración educativa, por lo menos no en Canarias. El «Proyecto clic Escuela 2.0» dejó no sólo las tecnologías en el aula sino también muchas prácticas e iniciativas pedagógicas que han sido el germen y el aliciente para seguir trabajando en el uso pedagógico de las TIC.

En este sentido, actualmente en Canarias, la Consejería de Educación, Universidades y Sostenibilidad (CEUS) continúa implantando y desarrollando iniciativas de apoyo para la integración de las TIC en todos los contextos educativos (administración, centros y aulas) y dirigidas a toda la comunidad educativa. Estas iniciativas, en este periodo postEscuela 2.0, están representadas por las siguientes acciones institucionales:

a) Se potencia el «Portal ECO Escuela 2.0», creado en el contexto del «Programa clic Escuela 2.0», como entorno virtual de trabajo cooperativo y colaborativo para los centros y el profesorado, y con la finalidad de visualizar las prácticas educativas con TIC. Se dinamiza su uso poniendo en funcionamiento nuevos espacios y herramientas con la finalidad de impulsar el cambio metodológico en los procesos de 
enseñanza y aprendizaje y el desarrollo de la competencia digital e informacional de toda la comunidad educativa. Así dentro del Portal ECO se crean un entorno virtual para que el profesorado gestione su propia formación a partir de la oferta formativa presencial y on line, además de un espacio de autoformación a través de una variedad de materiales y recursos digitales. Se oferta también un repositorio de recursos digitales creados por profesorado, por colectivos, desde proyectos o programas institucionales y organizados en una variedad de categorías; así también ejemplos de situaciones de prácticas organizadas por etapas educativas, y de ejemplos de buenas prácticas, además de otros servicios de interés como el acceso de los Centros de Profesores (CEP) y a la red educ@contic-inf. Destacan los servicios y espacios para la creación de blogs a centros y profesorado como estrategia para visualizar, compartir las prácticas educativas e informar y comunicarse con la comunidad educativa.

Como sistemas de apoyo a los centros educativos y profesorado en la utilización del Portal ECO Escuela 2.0 se potencian los dos servicios que ya existían, como son el Portal Medusa, como apoyo pedagógico, y el Centro de Atención al Usuario (CAU_CE) como apoyo técnico. Como novedad se crea el Área de Tecnología Educativa (ATE), Medusa queda integrada en dicha Área.

b) La creación de un nuevo proyecto institucional para la integración de las TIC, el Proyecto TSP o Tecnologías al Servicio de las Personas y Gestión del Conocimiento, y cuyo ámbito de actuación son los centros de Educación Primaria y Secundaria. Con este proyecto se continúa con la línea del modelo 1:1 en el sentido de que contempla las tecnologías en las aulas, y por tanto, la creación de las condiciones óptimas de accesibilidad y conectividad.

Los objetivos y estrategias del proyecto se orientan tanto al desarrollo de las condiciones tecnológicas como pedagógicas necesarias para que las denominadas tecnologías emergentes e innovadoras se visualicen y sean accesibles para la comunidad educativa, de forma que se integren con naturalidad en las prácticas educativas y lo llamativo sea «lo que se hace» con las tecnologías y no las tecnologías en sí mismas. En este sentido, se plantean desde la actualización y renovación de las dotaciones existentes a la implantación de aulas digitales en los niveles de Educación Infantil, Primaria y Secundaria Obligatoria; la revisión, actualización e instalación de infraestructura de redes a la instalación de servidores, periféricos y otro hardware que mejore la accesibilidad y conectividad. Como objetivos y estrategias destinadas a favorecer la integración pedagógica de estas tecnologías, además de dinamizar las que ya se están desarrollando en el Portal Eco escuela 2.0, destacan entre otras, las acciones orientadas a la investigación y experimentación de las tecnologías emergentes aplicables en los procesos educativos y la definición de posibles estrategias de transferencias a las aulas; y la implantación de herramientas y modelos de gestión de la información y del conocimiento en un contexto de innovación e investigación educativa con la participación de alumnado, profesorado y otros agentes educativos.

El TSP, como proyecto institucional de innovación educativa con TIC, se plantea también objetivos y estrategias destinadas, por un lado, a comunicar y dar a conocer a la comunidad educativa las finalidades y actuaciones del proyecto; y por otro, el diagnóstico de la integración de las tecnologías digitales en los centros y aulas y el seguimiento de los efectos de la implantación progresiva del proyecto. En la actualidad se está llevando a cabo la evaluación diagnóstica de la integración de las TIC en la educación escolar, bajo la responsabilidad del Laboratorio de Educación y Nuevas Tecnologías de la Universidad de La Laguna (EDULLAB).

\subsection{Cataluña}

Los proyectos «eduCat 1x1» (2009-2011) y «eduCat 2.0» (2011-12), la concreción y materialización en Cataluña del programa Escuela 2.0, llegan a las aulas y a los centros educativos de esta comunidad 
autónoma tras casi 30 años de políticas encaminadas a promover la utilización de las Tecnologías de la Información y la Comunicación (TIC) en los procesos de enseñanza y aprendizaje (Alonso, 2012).

En el marco de estos proyectos («eduCat 1x1» y «eduCat 2.0»), la administración educativa catalana, se propuso 4 grandes líneas de actualización: (1) digitalizar las aulas (de forma generalizada en los primeros cursos de Secundaria y puntualmente en algunos centros de Primaria); (2) garantizar la conectividad de todos los centros de secundaria; (3) promover el acceso a libros de texto y a otros contenidos digitales; y (4) ofrecer formación y asesoramiento al profesorado.

Sin lugar a dudas, el cableado de los centros, la conectividad (wifi), el suministro de PDI y la cofinanciación masiva (a partes iguales entre la administración y las familias) de ordenadores portátiles para los alumnos, fueron en su momento las actuaciones más mediáticas y son, en la actualidad, las huellas más visibles de los proyectos «eduCat 1x1» y «eduCat 2.0». Aproximadamente, el 85\% de los centros de secundaria en Cataluña decidieron, voluntariamente, participar en el proyecto «eduCat 1x1», y cuentan en la actualidad con un nivel de equipamientos e infraestructuras que podríamos calificar de bastante satisfactorio. En el 15-20 \% de centros que no se adhirieron, es notorio un cierto déficit de infraestructuras tecnológicas que se intentó compensar en el último ejercicio presupuestario con la dotación de PDI. El curso 2011-12, los responsables del programa Escuela 2.0, sugieren a la administración educativa catalana que comience sus actuaciones en los últimos cursos de primaria, ya que prácticamente se había conseguido uno de los objetivos más emblemáticos del proyecto: que todos los estudiantes de primer y segundo curso de la ESO tuviesen un ordenador portátil en propiedad. Pero la repentina suspensión del programa Escuela 2.0, paraliza las actuaciones en materia de equipamientos e infraestructuras en las escuelas de Primaria. En el mes de junio de 2012, desde el Departament d'Ensenyament, se envía a los centros una circular en la que se informa de la nueva situación: la supresión de la dotación presupuestaria por parte de la administración central para la adquisición de equipamientos informáticos y libros digitales. En este mismo documento, la administración autonómica ofrece a los centros su asesoramiento y una serie de orientaciones, criterios y especificaciones técnicas a tener en cuenta a la hora de seleccionar los dispositivos digitales personales de los alumnos.

En estos momentos, son muchos los centros educativos catalanes que continúan apostando por los modelos 1:1 desde múltiples interpretaciones. Unas lecturas que van desde aquellos centros en los que todos los alumnos de primer curso de la ESO tienen que comprar un ordenador portátil (las familias abonan el $100 \%$ de su importe) y llevarlo cada día a clase, a aquellos otros que consideran que el modelo anterior no es un modelo sostenible (los portátiles son propiedad de las familias y la inversión de la administración no ha repercutido en la optimización del equipamiento informático de los centros) y apuestan porque los ordenadores portátiles sean propiedad del centro, a partir de la financiación de las AMPAS o las administraciones locales (ayuntamientos), entre otras instituciones y organismos.

En relación a la tercera línea de actuación del proyecto «eduCat 1x1», promover el acceso a libros de texto y a otros contenidos digitales, desde la administración se asignaron 35 euros por alumno y año para la adquisición de libros de texto digitales gestionada a través de la plataforma Atria, un portal que, coincidiendo con la supresión del programa Escuela 2.0 y consecuentemente los proyectos «eduCat $1 \times 1$ » y «eduCat 2.0», tuvo que detener su operativa comercial, tras una sentencia judicial, a finales del mes de marzo de 2012, al entrar en un conflicto de intereses con el sector editorial. En estos momentos, la situación se ha normalizado gracias a la puesta en funcionamiento del proyecto Marsupial, una plataforma que se utiliza en todo el estado español y que recientemente ha comenzado a implementarse en América latina. Este portal define unos protocolos de comunicación entre las diferentes plataformas editoriales, garantizando la interoperatividad y posibilitando que los centros accedan a contenidos editoriales de pago y a contenidos libres o en abierto, indistintamente y de forma integrada en una misma 
plataforma. En estos momentos, existe una nueva propuesta del Ministerio de Educación, el «Punto Neutro», un portal próximo a lo que fue Atria y que parece no haber encontrado ningún tipo de acogida entre los responsables de las políticas TAC (Tecnologías del Aprendizaje y el Conocimiento) en Cataluña ni entre las editoriales del sector. Finalmente remarcar que responsables del Área TAC nos manifestaron que aproximadamente un $20 \%$ de los centros de enseñanza secundaria catalanes utilizan únicamente libros de texto digitales y que prácticamente un $50 \%$ de los centros, utilizan los libros de texto digitales y los libros en soporte papel.

En el marco de la cuarta línea de actuación, la formación y asesoramiento del profesorado, es posible constatar que la formación telemática individual que se había venido desarrollando desde la administración, ha disminuido considerablemente en estos momentos. En cierto modo podríamos hablar de un cambio de modelo «buscado» que prioriza la formación interna orientada a proyectos específicos y singulares de centro. Destacar también la red EduCat, una red social dentro de Ning que sirve como espacio de comunicación y como plataforma para el diseño y la organización de talleres abiertos, unas actividades que no están reglamentadas, que no cuentan con una certificación final y que parten de los intereses y del trabajo colaborativo entre docentes. Se trata de una modalidad que se mueve entre el concepto de MOOC y el de Comunidad de Práctica. Finalmente remarcar que en estos momentos la administración educativa explicita la necesidad de contar con docentes competentes digitalmente (Alonso, Guitert y Romeu, 2014) capaces de traspasar esta competencia al alumnado. Con esta finalidad, desde la administración se ha abierto y provocado un debate en el que se pone de manifiesto la necesidad de colaboración y complicidad de las universidades catalanas en la formación inicial del profesorado en el ámbito de las competencias digitales

En una conversación mantenida recientemente con algunos de los responsables del Área TAC del Departament d'Ensenyament de la Generalitat de Catalunya, éstos manifestaron que tras la finalización del programa Escuela 2.0, la administración educativa catalana, ante la imposibilidad de continuar destinando partidas presupuestarias para la dotación de infraestructuras y equipamientos en los centros, opta por focalizar sus actuaciones en cuestiones mucho menos tangibles pero más pedagógicas, como son: los conceptos, las ideas, las metodologías, los materiales y los recursos, las orientaciones, la formación del profesorado, las sugerencias y recomendaciones.

En coherencia con lo que acabamos de afirmar, en reiteradas ocasiones, la actual consejera de enseñanza, ha manifestado que el programa Escuela 2.0 focalizaba la mirada en un uso de la tecnología como canal de distribución de contenidos digitales (aprender de la tecnología). Partiendo de este presupuesto, desde el curso 2011 la administración realiza una apuesta por el desarrollo de las competencias digitales en los alumnos y una utilización de los dispositivos tecnológicos que promuevan su crecimiento digital. En esta misma línea, en el periodo 2011-2014, desde la administración, se ha apostado por el diseño y la singular implementación del Plan TAC de Centro o un Proyecto Curricular de Centro que tiene como objetivo último una visión integral de las tecnologías en el centro y que persigue el desarrollo de las competencias digitales del alumnado en un centro competente digitalmente.

Finalmente hemos de señalar que algunas de las actuaciones que en estos momentos se están implementando desde el Área TAC del Departament d'Ensenyament de la Generalitat de Catalunya son las que siguen:

- Desde hace dos años, el Departament d'Ensenyament viene participando en el programa $m S c h o o l s{ }^{9}$, una iniciativa de "partenariado» o colaboración pública/privada que impulsa la Fundación Mobile Word Capital Barcelona, en colaboración con la Generalitat de Catalunya, el Ayuntamiento de Barcelona y la Asociación GSM (organización de operadores móviles y

\footnotetext{
9 http://www.xtec.cat/web/projectes/mschools
} 
compañías relacionadas, dedicada al apoyo de la normalización, la implementación y promoción del sistema de telefonía móvil GSM). El proyecto mSchools tiene 3 ejes de actuación: (1) incorporar las tecnologías móviles en un sentido amplio (dispositivos móviles de uso personal) en los procesos de aprendizaje; (2) potenciar las competencias digitales y la emprendeduría de los estudiantes; y (3) crear un entorno abierto para la «mEducación». Estos tres ejes se han ido concretando en diferentes proyectos:

- Mobilitzem la Informàtica ${ }^{10}$, un proyecto orientado a los alumnos de $4^{\circ}$ curso de la ESO cuyo objetivo es repensar la asignatura optativa de informática, es el proyecto que ha tenido más impacto mediático. En el marco de este proyecto, los alumnos identifican un problema que pueda ser resuelto con una aplicación del teléfono móvil, y a partir de aquí, diseñan las apps, hacen el prototipo y elaboran una propuesta de comercialización. Aprenden a programar para resolver un problema y generar un producto que tiene que ser sostenible y tener una salida comercial. Un elemento a destacar de este proyecto es la colaboración con las empresas. Se solicitaron voluntarios que desde las empresas pudiesen asesorar presencial o virtualmente los diferentes proyectos en dos o tres momentos a lo largo del curso académico. En estos momentos se cuenta con 150 voluntarios de empresas del sector. A final de curso se seleccionan las mejores apps y se celebra un concurso con stands, jurado (algunos miembros de compañías extranjeras), premios, trípticos, merchandising... en el que los alumnos, muchos de ellos en inglés, deben intentar comercializar su producto. El curso pasado participaron en este proyecto 200 centros y 6000 alumnos, y este curso se ha doblado la cifra de alumnos participantes.

- Mobile Learning Awards ${ }^{11}$, el objetivo de esta modalidad es premiar experiencias innovadoras de uso educativo de los dispositivos móviles que muestren el potencial de estos dispositivos para el aprendizaje, la organización, la convivencia o la comunicación con las familias. El curso pasado se presentaron unas 70 experiencias que contribuyeron a dar visibilidad y reconocimiento a las iniciativas.

- Mobile History Map $(M H M)^{12}$, un proyecto pionero, que acaba de ponerse en marcha este curso escolar, y que potencia el trabajo colaborativo con soluciones móviles en el ámbito de las Humanidades. Esta iniciativa parte de una nueva aplicación móvil de geolocalización que permite a los alumnos crear contenidos (en cuatro idiomas) relativos a puntos de interés próximos a su centro educativo (patrimonio monumental, cultural, histórico, gastronómico, etc.)

\subsection{Comunidad de Madrid}

La Comunidad de Madrid heredó a finales de los años noventa el programa de tecnología educativa nacional. Desde entonces implementa la integración en los centros mediante dotaciones, formación y programas experimentales. Como se dijo en un principio, decidió no participar en el programa Escuela 2.0 y poner en marcha uno propio.

El Programa piloto de Institutos de Innovación Tecnológica de la Comunidad de Madrid es su principal propuesta. Quiso diferenciarse sensiblemente e incumplía buena parte de los principios clave del modelo 1:1. Los ordenadores estaban sólo en las aulas, participaban niños de secundaria, no había un plan regional de conectividad de las escuelas y hogares y se organizó con un software propietario en terminales en red anclados al suelo de las aulas, dispuestos en hileras. Las ideas de excelencia y competencia sustentan además una visión elitista del Programa. Los participantes deben asumir una

\footnotetext{
${ }^{10}$ http://alexandria.xtec.cat/

${ }^{11}$ http://www.xtec.cat/web/centres/alscentres/premis/mla2014

${ }^{12}$ http://www.xtec.cat/web/projectes/mschools/mhm
} 
pedagogía del esfuerzo que se debe visibilizar en una detallada evaluación de los resultados del Programa.

Tras cuatro años, el programa pervive en los 15 Institutos de Innovación Tecnológica, como centros piloto (sobre los más de 300 públicos de secundaria de la región), con sus dotaciones (que se han completado hasta $4^{\circ}$ de la ESO), la política de formación en el centro y los incentivos personales (que se mantienen congelados desde 2010).

Se realizan reuniones en seminarios y grupos de trabajo para la puesta en común y nuevos desarrollos (recientemente un taller de 100 profesores del programa donde se construyó un banco de cursos en Moodle), y se aprovecha la experiencia del programa para apoyar otros programas (el de tabletas de la fundación Idea y el de institutos de alto rendimiento deportivo, que se mencionan más adelante). También hay una red informal de profesores del programa.

Son estos Institutos los que ponen en marcha la experimentación de una nueva optativa del currículo de educación secundaria denominada «Programación». La asignatura se implanta de forma progresiva en 2014-15 en estos Institutos. En el curso 2015-16, se extiende a todos los centros en Primero y Tercero de la ESO. En 2016-17, a Segundo y Cuarto de la ESO. Para formar al profesorado en esta nueva asignatura la Comunidad de Madrid, en colaboración con Telefónica Learning Services, ha puesto en marcha «Code Madrid» ${ }^{13}$. Se trata de una propuesta para formar a 1.500 profesores en un año con tres MOOC de 50 horas de formación cada uno, diez de ellas presenciales en los centros de formación del profesorado (Comunidad de Madrid, 2014).

La Comunidad de Madrid ha puesto en marcha proyectos de excelencia similares a éste pero en otras temáticas, como es la del alto rendimiento deportivo (5 institutos). Han recurrido a la experiencia de los Institutos de innovación tecnológica, aunque en menor escala, para apoyar estas otras experiencias con tecnología. Por otra parte, se ha puesto en marcha un proyecto piloto de saturación tecnológica con tabletas, experimentado desde 2011 en dos institutos madrileños y uno murciano.

Los objetivos que declara el consorcio del proyecto, denominado Idea, son: mejorar los resultados de los estudiantes, normalizar la digitalización de la enseñanza, aligerar las mochilas de los estudiantes, sencillez en el manejo, sobre todo en el caso de los profesores y de los padres; que proporcione un acceso organizado y estructurado a los contenidos educativos de calidad existentes en la red; que sea viable tanto técnica como económicamente, con contenidos personalizables, un proceso de aprendizaje cuantificable, y un modelo que consiga captar de forma más rápida y eficiente la atención, interés y compresión.

El proyecto es promovido por la Fundación Albéniz y financiado por la Comunidad de Madrid, la editorial Pearson y la empresa Samsung (Samsung School). Un servicio web de distribución de recursos educativos digitales, orientado a satisfacer las nuevas exigencias de los mercados surgidas en torno al desarrollo de la escuela $2.0^{14}$. Los centros educativos deben facilitar conectividad y los padres adquirir los materiales (al punto de que se convertirá en un proyecto BYOD, «Trae tu propio equipo»).

El proyecto ofrece a los padres una tableta, donde tienen metidos libros de seis materias, agenda con deberes, avisos de exámenes, un apartado de información del profesor a los padres y los horarios.

«Es una base tecnológica en la que cualquier editorial puede colocar sus libros; cada colegio, cada profesor, elige, y después los padres lo compran; igual que con los libros de papel. Las familias, cuando abonan el libro, pagan también todo lo demás, la plataforma y el

\footnotetext{
${ }^{13}$ http://gestiondgmejora.educa.madrid.org/codemadrid/

${ }^{14}$ http://www.fundacionalbeniz.com/F_Proyecto_idea.aspx
} 
mantenimiento (cada editorial llega a un acuerdo con iDEA para colocar sus textos en el sistema $)^{15}$

La tableta también la compran los padres. En algún caso el coste de los libros es un tercio de los de papel (si la compra la realiza la asociación de padres) ${ }^{16}$. Hay una distribuidora de libros de texto ${ }^{17}$ que facilita el acceso ubicuo a los materiales a los estudiantes, la posibilidad de fabricar materiales complementarios a los profesores, así como tareas de gestión (valoración de actividades de los estudiantes, seguimiento de los padres). En clase se utiliza una tecnología nube multipantalla (tabletas y portátiles, pizarra digital.)

«que permite el trabajo sin conexión y la sincronización de los contenidos. La herramienta ofrece un software sencillo que se organiza en torno al libro de texto digital, considerado el elemento esencial que garantiza la calidad de la enseñanza y normaliza las dinámicas de trabajo, que también integra un banco de recursos, con contenidos educativos que aporten valor añadido, así como herramientas para la gestión académica por parte del profesor y para la comunicación con alumnos, padres y equipo directivo del centro» ${ }^{18}$.

La evaluación realizada de la experiencia habla de un alto porcentaje de satisfacción entre profesores y estudiantes, participación en clase, trabajo autónomo y mejora en estrategias de búsqueda de información. Algunos profesores añaden que con el proyecto se identifica colaboración, inmediatez de feedback y trabajos de investigación de los alumnos, aunque hay problemas para controlar las aulas, lo que motiva que se haya pedido ayuda a los centros que experimentan el programa de Institutos de innovación tecnológica. Los centros valoran que ofrece a los padres la oportunidad de participar adquiriendo una nueva responsabilidad en lo referente al uso adecuado del dispositivo digital como herramienta de trabajo.

En la puesta en marcha del proyecto hubo problemas de conectividad. Con la tableta hay problemas de roturas, robos, pérdida, como ocurre en otros programas, aunque en la experiencia de éste no ha tenido demasiadas incidencias. Los docentes reclaman más acceso de los padres a la información que genera y mejoras en las herramientas administrativas ${ }^{19}$. Algunos profesores dudan de la utilidad social del proyecto, del excesivo interés en el mercado de libros de texto más que en el aspecto educativo, el abaratamiento tan limitado de los libros y las dificultades que abre a las familias con menos recursos para disponer de las tabletas.

Otras iniciativas de la Comunidad de Madrid están orientadas a la introducción de Smartphone y tabletas en otros ámbitos. Por una lado, en la formación online pública, para niños con discapacidad intelectual, desde el centro regional de innovación y formación Las Acacias, en colaboración con la Fundación Vodafone España y la Fundación Garrigou. Se ha dado formación a 100 profesores.

Por otra parte se ha apoyado con fondos públicos un Programa de inserción laboral para jóvenes en situación de más dificultad o en riesgo social, organizado por la Federación de Plataformas SocialesPinardi, una entidad vinculada a la orden salesiana, relacionada tradicionalmente a la formación profesional, impulsando en este programa actividades relacionadas con la Formación Profesional Básica.

\footnotetext{
${ }^{15}$ http:/ / sociedad.elpais.com/sociedad/2013/04/28/actualidad/1367172178_824579.html

${ }^{16}$ http://www.educa.madrid.org/web/ies.santamarca.madrid/novedades/librosdigitales2015a.pdf

${ }^{17}$ https://www.blinklearning.com

${ }^{18}$ http://blog.universoidea.com/tag/tablet/

${ }^{19}$ https://www.youtube.com/watch?v=lTVdNa4-GQ8
} 


\subsection{Comunidad Valenciana}

La Conselleria de Educación de la Comunidad Valenciana no se adhiere en 2009 al Programa Escuela 2.0 del Ministerio de Educación. Como alternativa pone en marcha un proyecto propio, el «Centro Educativo Inteligente» (CEI) cuyo objetivo es integrar las TIC en la totalidad de los espacios existentes en los centros escolares públicos de los niveles no universitarios. El plan arrancó experimentalmente en dos centros, uno de Primaria y otro de Secundaria, y pese a las imprecisiones del modelo propugnado, en el curso 2010-2011 participan en el proyecto CEI 18 centros educativos: 7 de Secundaria, 9 de Infantil y Primaria, 1 Centro de Acción Educativa Singular y 1 Centro Rural Agrupado.

Al curso siguiente, la administración se propuso extender este proyecto a más de 70 en una segunda fase. Se iniciaron las gestiones, así lo atestiguan directores de centros y algún que otro documento inacabado, aunque no se llegó a implementar, fundamentalmente por la escasez de recursos debido a los recortes presupuestarios en educación.

Se ha creado en el programa una comunidad de «Centros Educativos Inteligentes» que dispone de una plataforma de acceso privado en el portal educativo de la Conselleria de Educación. Uno de sus objetivos es potenciar el intercambio de experiencias docentes e impulsar nuevas vías de comunicación entre ellos y con la Administración educativa. Los recursos disponibles son propios y los materiales en abierto son los que se encuentran en la propia web de $«$ Mestre a casa ${ }^{20}$, plataforma creada algún tiempo antes de ponerse en marcha los CEI.

En el trabajo de campo realizado, llama la atención que más de un tercio del profesorado encuestado de los CEI, manifiesta estar de acuerdo con esta iniciativa de la Administración autonómica, especialmente por lo que supone de mejora del equipamiento tecnológico de los centros. Sin embargo, en una proporción parecida muestran su desacuerdo al rechazo desde la Generalitat a la propuesta del ministerio sobre la Escuela 2.0.

Estas reticencias hacia la decisión adoptada, tal vez sea un anticipo de lo que finalmente ha pasado con todo el despliegue que rodeó a los CEI. Como nos decía el director de un centro, durante dos años fue el anfitrión ineludible de cuantas delegaciones extranjeras llegaban a la Conselleria, pues para todas era «obligada» la visita a uno de los centros piloto CEI. Sin embargo, en 2012 el equipo responsable de la iniciativa CEI y demás tecnologías para los centros, fueron desplazados a la Dirección General de Tecnologías de la Información adscrita ahora a la Conselleria de Hacienda y Administración Pública. Y desde esta instancia se han diseñado proyectos como:

- Plan Estratégico de Innovación y Mejora de la Administración de la Generalitat 2014-2016 ${ }^{21}$

- Agenda Digital de la Comunitat Valenciana ${ }^{22}$.

En resumen, apenas quedan vestigios del proyecto CEI en las webs de la administración autonómica. No obstante, en la web de la Conselleria de Hacienda existe una pestaña a la plataforma «Mestre a casa» y, desde ésta, otra que nos ofrece una breve presentación de los CEI. Estos enlaces son, de todos modos, los mismos que aparecen en el servicio de Innovación Tecnológica Educativa adscrito a la Conselleria de Educación ${ }^{23}$.

Por otro lado, no hay ninguna evaluación oficial del mismo ni informes que den cuenta de lo sucedido y logrado con este proyecto. La prensa ha comentado el informe que en 2013 hizo una

\footnotetext{
${ }^{20} \mathrm{http}: / /$ goo.gl/g6Frf

${ }^{21} \mathrm{http}: / /$ goo.gl/pXDwRz

${ }^{22}$ http://goo.gl/Ovwg27

${ }^{23}$ http://goo.gl/1nBjzS
} 
consultora de comunicación (Tertulia Digital), pero las críticas a la metodología utilizada fue tan cuestionada, que lo han retirado.

En la actualidad se potencia el uso de libros de texto en tabletas digitales en centros educativos sostenidos con fondos públicos. Las dos convocatorias realizadas hasta la fecha, se rigen por las siguientes normas: Resolución de 10 de junio de 2013, de la Dirección General de Innovación, Ordenación y Política Lingüística (DOCV 7047 / 17.06.2013) y la Orden 63/2014, de 28 de julio, de la Conselleria de Educación, Cultura y Deporte (DOCV 7330 / 01.08.2014).

El objetivo general del programa es fomentar el uso de libros de texto digitales utilizando las tabletas como soporte físico. Mientras que los objetivos específicos son:

- Experimentar una dinámica de enseñanza en la que se incorpora la tableta digital como soporte para el libro de texto.

- Experimentar una alternativa a la adquisición de libros de texto en soporte papel como medida de ahorro para las familias.

- Ayudar a determinar la correlación entre el uso de las TIC en el aula y una mejora del proceso de enseñanza-aprendizaje.

- Evaluar el potencial uso de las tabletas digitales en la búsqueda de una educación de calidad y adaptada a la diversidad.

- Valorar las limitaciones del modelo.

El plan piloto del programa se pone en marcha en el curso 2013-2014, en el quinto curso de Primaria de los centros públicos y concertados acogidos en la convocatoria, y con el propósito de ampliación en el siguiente a sexto y a otros nuevos centros, si los resultados fueran favorables. Los contenidos utilizados en las tabletas digitales podían provenir de editoriales o bien ser contenidos propios elaborados por el profesorado. Ambas modalidades no eran excluyentes, de modo que en un mismo centro educativo se podían utilizar las dos. En total, en el primer año participaron un total de 1000 alumnos de veinticuatro centros educativos de la Comunidad Valenciana, nueve pertenecientes a la provincia de Alicante, doce a la de Valencia y tres a la de Castellón ${ }^{24}$. La segunda convocatoria, curso 2014-2015, se ha ampliado el número de centros hasta los 49, con un total de 3000 estudiantes de $5^{\circ}$ y $6^{\circ}$ de Primaria. De ellos 7 pertenecen a la provincia de Castellón, 20 de Alicante y 22 de Valencia ${ }^{25}$.

En la primera convocatoria se puso a disposición de los centros y de las familias una plataforma unificada de acceso a los contenidos de las distintas editoriales (Blinklearning) que participaban en el programa (Oxford University Press, Edelvives, Anaya, SM, Bromera, Casals, MacMillan, Tàndem, Edebé, Pearson, Cambridge y Santillana). Sin embargo, esta iniciativa despertó diversas críticas entre el mundo editorial, los centros y las familias, tal y como se expresaba el coordinador TIC de uno de los centros: «Eso no era una página, era un mercado» (ECT1, 578).

En la segunda convocatoria, esta iniciativa ha desaparecido y en su lugar ha aparecido Llibrey ${ }^{26}$, otra web que la Conselleria ha puesto a disposición de las familias para facilitar el intercambio de libros de texto, no sólo digitales, entre el alumnado de toda la Comunidad. Iniciativa denunciada por la Asociación Nacional de Editores de Libros y Material de Enseñanza (ANELE) por vulnerar la libre competencia y los intereses comerciales de sus miembros ${ }^{27}$.

\footnotetext{
${ }^{24}$ Levante-EMV (2014). Educación extenderá el programa de tabletas a sexto de Primaria. Recuperado de: http://goo.gl/o4ikm4

${ }^{25}$ http://goo.gl/Mxyfg4

${ }^{26}$ http://llibrey.edu.gva.es/

${ }^{27}$ http://goo.gl/xkdBJ0
} 
Cabe destacar que la repercusión económica del programa para la administración educativa es prácticamente nula. Pues los padres están obligados a asumir los gastos del soporte y de los contenidos curriculares digitalizados (lo que ha generado no pocos conflictos en las AMPAs este comienzo de curso) y los centros la infraestructura tecnológica necesaria (Internet, Wifi, cableado...).

\subsection{Extremadura}

La Consejería de Educación y Cultura del Gobierno de Extremadura (2013) evaluando los últimos diez años, consideró que se ha realizado un importante esfuerzo para conseguir el uso de real y cotidiano de las $\mathrm{TIC}^{28}$. El conjunto de actuaciones de anteriores gobiernos regionales es descrito como un «ambicioso despliegue tecnológico» que ha conducido a disponer de una amplia dotación informática en los centros educativos. Se distinguen dos modelos de intervención diferenciados: «TIC en la escuela» (1995-2001) y «TIC en el aula» (2001-2011) y se aboga por iniciar un nuevo período que dé lugar a las «Tecnologías de la Educación en la Comunidad Educativa». En consecuencia, se aprueba el «Plan de Acción de las Tecnologías de la Educación» denominado «Comunidad Educativa 2.0».

\section{eScholarium}

En octubre de 2013 se presenta oficialmente una nueva plataforma educativa o «Entorno Virtual de Educación para Enseñanzas no Universitarias», denominada «eScholarium», que se encuentra encuadrada dentro del Programa Estratégico de TIC Educativas de Extremadura (ITER) y como plan de la acción «Comunidad Educativa 2.0». El propósito de esta plataforma educativa es facilitar el acceso y adquisición de contenidos educativos y libros de texto digitales, libres o de pago, y el posterior seguimiento de la relación que establecen alumnado y profesorado a través de estos contenidos. eScholarium se concibe como un servicio público que está alojado en servidores de la Consejería de Educación y Cultura para garantizar la protección de datos, la autonomía, el acceso por intranet y la neutralidad (no se favorece a ningún editorial). La plataforma es accesible a través de múltiples dispositivos y está adaptada a diferentes sistemas operativos. Incorpora libros de textos digitales de cualquier editorial que cumpla los estándares «marsupial» ${ }^{29}$. El uso está adaptado tanto a no-lectores como neo-lectores y lectores. La plataforma está integrada con Rayuela, un sistema integrado para la comunicación y la gestión educativa (acceso de credenciales; carga de grupos, tutores y profesores; faltas de asistencia; calificaciones). Tiene un sistema off-line que permite su utilización sin conexión a Internet en determinados momentos y para algunas actividades. El servicio incluye la atención al usuario (eSchoCAU) para atender dudas e incidencias por teléfono, correo electrónico y web, ofrecer formación y evaluar la satisfacción de los usuarios. En el curso 2013-14 se realizó un primer pilotaje con libros digitales en ordenadores portátiles y para el curso 2014-15 se han incorporado más centros, aplicando el modelo BYOD (Bring Your Own Device).

Los objetivos más relevantes de «eScholarium» son los siguientes:

- Poner al servicio de toda la Comunidad Educativa Extremeña, una aplicación web que permita la realización, desarrollo y seguimiento de actividades de enseñanza-aprendizaje en las modalidades presencial, semi-presencial y a distancia, de enseñanzas no universitarias.

\footnotetext{
${ }^{28}$ Instrucción 25/2013 de 18 de Septiembre de 2013. Consejería de Educación y Cultura. Gobierno de Extremadura.

${ }^{29}$ El proyecto Marsupial (3\&Punt - Soluciones Informáticas) está orientado a la realización de los módulos necesarios para que desde un LMS (Moodle) se pueda acceder de una manera autenticada a los contenidos situados en los servidores de los proveedores de los contenidos y posteriormente guardar e integrar en el LMS los resultados de la interacción con dichos contenidos. El proyecto contempla dos tipos de contenidos: SCORM 1.2 y contenido web. Para más información: http://es.scribd.com/doc/39428841/Marsupial
} 
- Potenciar un modelo de educación virtual apoyado en las TIC, que se adapte a las condiciones personales y laborales de los individuos (en cualquier sitio y en cualquier momento) y a las características peculiares de nuestro territorio.

- Promover y colaborar en la adopción de conceptos modernos que caracterizan nuestro modelo TIC educativo:

- Permitir el uso de contenidos educativos y libros de texto libres y de pago, en formato digital.

- Aprovechar así óptimamente los recursos actuales de las aulas tecnológicas.

- Permitir desarrollar procesos de enseñanza-aprendizaje sincrónicos y asíncronos a través de la red.

- Apoyar la labor docente con un «Motor de Asistencia Pedagógica Inteligente» (MAPI).

- Promover la creación y/o adaptación de contenidos educativos digitales por parte del profesorado de la Comunidad Extremeña.

- Pero también colaborar en el desarrollo y puesta en producción de software y servicios web y participar en redes sociales de interés educativo.

- Impulsar la World Wide Web como plataforma de trabajo, de formación, de información y de comunicación, en especial, con herramientas propias de la web 2.0.

- Facilitar la relación entre, por una parte, el conjunto de usuarios de la comunidad educativa con las diversas editoriales, empresas o autores que produzcan y comercialicen contenidos y aplicaciones digitales educativos, de pago o gratuitos.

La plataforma es la misma para todas las áreas y miembros de la comunidad educativa y está adaptada específicamente a las enseñanzas no universitarias. Incluye el uso de técnicas de learning analytics y es funcional en todos los tipos de dispositivos y sistemas operativos. Se integra con Rayuela, los repositorios de contenidos educativos digitales nacionales y regionales y otros servicios educativos, para formar una herramienta integrada de los recursos profesionales del docente.

Portfolio de la Competencia Digital Docente (CDD)

La Consejería de Educación del Gobierno de la Comunidad Autónoma de Extremadura ha desarrollado el «Portfolio de la Competencia Digital Docente (CDD)» (Pulido, 2014) que ofrece una serie de indicadores para que el docente pueda conocer su nivel competencial; los conocimientos, destrezas y actitudes que deben desarrollar para alcanzar un determinado objetivo competencial y cuáles son los itinerarios formativos para alcanzarlos. La CDD está constituida por un conjunto de conocimientos, destrezas y actitudes que permiten, por una parte, usar de modo eficiente en el ámbito educativo los diferentes dispositivos tecnológicos vinculados a la enseñanza; las aplicaciones y servicios digitales de gestión y comunicación; así como los contenidos digitales vinculados con las áreas y materias curriculares. Por otro lado, permiten utilizar las estrategias adecuadas para promover en el alumnado el desarrollo de su propia competencia digital.

El Portfolio de la CDD también utiliza las cinco áreas competenciales del Marco Europeo de la Competencia Digital Ciudadana y añade una sexta área que denomina «Aplicabilidad al ámbito educativo». Además, define tres niveles competenciales: (a) Básico o inicial (niveles A1 y A2), orientado hacia el conocimiento de las TIC y su aplicación docente; (b) Medio o de progreso (niveles B1 y B2), orientado al conocimiento y uso de las TIC y su aplicación a la enseñanza y (c) Avanzado o final (niveles C1 y C2), orientado a la elaboración de materiales didácticos, trabajo colaborativo en red, difusión de buenas prácticas. Cada área competencial incluye 5 dimensiones descriptivas: denominación, 
competencias,niveles de dominio, ejemplos de conocimientos, destrezas y actitudes, así como la aplicabilidad en el aula.

\subsection{País Vasco}

Aunque desde el último cambio político ha habido interés en la Comunidad Autónoma del País Vasco de finalizar con las iniciativas del programa «Eskola 2.0», hay que reconocer que el programa ha servido para dinamizar la formación del profesorado, promover la reflexión sobre muchas iniciativas de cambio educativo asociadas con las tecnologías y dotar a los centros de recursos digitales (Losada, Karrera, \& Correa, 2011).

El principal poso que ha quedado de la experiencia «Eskola 2.0» es constatar la necesidad de un cambio metodológico dentro de las aulas, no solo como estrategia de integración de las tecnologías digitales sino como necesidad apremiante de una institución escolar que necesita un urgente cambio. La participación de los centros en el programa «Eskola 2.0» ha sido una oportunidad para repensar el quehacer docente y discente. Tampoco podemos olvidar que el programa «Eskola 2.0» converge con una variada gama de iniciativas que han venido a facilitar la expansión, a todo el ámbito curricular, de la reflexión sobre los aciertos, dificultades y carencias en la integración de las tecnologías digitales.

La iniciativa «Eskola 2.0» supuso un reto para el profesorado y generó necesidades de formación y desarrollo de nuevas competencias digitales, que iniciativas como «Formación 2.0», intentaron responder. En este sitio $\mathrm{web}^{30}$ se pueden ver un conjunto de recursos orientados a la formación del profesorado. Dividido en tres niveles (básico, medio y avanzado). En este otro ${ }^{31}$ podemos encontrar desde un pack básico de informática educativa hasta información sobre pizarras digitales o aplicaciones web 2.0.

La participación de las escuelas en proyectos de colaborativos han tenido un papel muy destacado en la integración y utilización de las TIC en los contextos escolares.

Entre estos proyectos colaborativos está el «Proyecto Pelutxe», para alumnos de Educación Infantil, con muy buena acogida en los centros escolares: Información sobre la puesta en práctica y organización del proyecto puede consultarse aquí32; para alumnos de $1^{\circ}$ y $2^{\circ}$ nivel está pensado el proyecto colaborativo «Asmakilo» ${ }^{33}$.

El proyecto «Gure herrietan zehar» ${ }^{34}$ ( $A$ través de nuestro pueblo), es un proyecto que pretende conocer mejor la realidad de cada pueblo y la utilización de las tecnologías digitales para la realización de diferentes tareas. Otros proyectos colaborativos interesantes son «Ni Kazetari» ${ }^{35}$ (Yo periodista), «Kosmodisea» $\mathrm{O}$ «Klik eta Klik».

«Eskola 2.0» ha dado un gran impulso a la organización de recursos digitales. Dentro de la iniciativa «Eskola 2.0» podemos consultar una web que funciona como centro de recursos y elaboración de materiales digitales ${ }^{36}$. En esta dirección tenemos acceso a recursos disponibles en Agrega, iniciativa a nivel estatal que merece la pena destacarse. También destaca dentro de los servicios consolidados el

\footnotetext{
${ }^{30}$ http://www.eskola20.euskadi.net/web/guest/prestakuntza20

31 http://www.eskola20.org/prestakuntza/laguntzatutoretzak/cursobasico/index.html

32 http://www.eskola20.euskadi.net/web/guest/peluche

$3 \mathrm{http}: / /$ www.eskola20.euskadi.net/web/guest/asmakilo

34 http://gureherrietanzehar.blogspot.com.es; http://www.eskola20.euskadi.net/web/guest/euskadin-zehar

35 http://www.eskola20.euskadi.net/web/guest/beste-proiektu-batzuk

${ }^{36}$ http://www.eskola20.euskadi.net/web/guest/eskola-2.0-baliabideak
} 
Servicio especial de familias y alumnado que pone a disposición de las familias recursos útiles para la educación de los hijos en materia digital ${ }^{37}$.

A principios del curso académico 2014/2015, aunque todavía activo, el protagonismo del programa «Eskola 2.0», está llegando a su punto final. La Resolución de 27 de junio de 2014, de la Viceconsejera de Educación de la Comunidad Autónoma del País Vasco, por la que se convoca a los centros públicos de Educación Primaria y de Secundaria Obligatoria de enseñanza no universitaria de esta Comunidad, a participar en el proyecto de innovación para la enseñanza-aprendizaje con recursos digitales, «Sare Hezkuntza Gelan», supone un punto de reorientación de la política de integración de las tecnologías digitales en las aulas y centros escolares.

Esta convocatoria ha seleccionado 25 centros escolares de diferentes niveles educativos, 10 de Educación Primaria y 15 de secundaria obligatoria. La finalidad de esta convocatoria es promover en los centros escolares participantes diferentes objetivos. Primero la elaboración de un proyecto de utilización de nuevas tecnologías y materiales y recursos digitales para el proceso de enseñanza aprendizaje en el aula. Segundo, la formación tecnológica y pedagógica del profesorado para la utilización de materiales y recursos digitales en el proceso de enseñanza aprendizaje. $Y$, por último, también tiene cabida en la convocatoria la creación de materiales digitales.

Las principales diferencias con el programa «Eskola 2.0» es la voluntariedad en la participación y la implicación abierta a los diferentes niveles educativos, no solo al alumnado comprendido entre los 10 y 14 años. «Sare Hezkuntza Gelan», insiste en la utilización de diferentes dispositivos tecnológicos y aprendizaje ubicuo. Pese a todo, el peso de las editoriales de libros de texto como facilitadores tecnológicos e impulsores de la renovación tecnológica en los centros al ofrecer dispositivos como las tablets, le puede estar haciendo un flaco favor a esta nueva iniciativa para la promoción de la tecnología en los centros escolares.

Las críticas hacia las limitaciones manifestadas por el programa «Eskola 2.0» se vuelven oportunidades para ensalzar las posibilidades del giro iniciado por la Consejería de Educación, hacia la utilización de otros tipos de dispositivos y recursos tecnológicos. Un giro tecnológico sustentado en la creencia que permitirá adaptarse a los diferentes ritmos de aprendizaje, impulsar el aprendizaje entre iguales, potenciar el aprender a aprender y ampliar el espacio físico temporal del aula en un entorno virtual y ubicuo de aprendizaje. Este nuevo programa no renueva el relato sobre las últimas tecnologías llamadas a habitar los espacios escolares, confiando en que la tecnología aportará el factor decisivo para conseguir la calidad educativa.

¿Pero este nuevo contexto de aprendizaje mediado por las nuevas invasiones tecnológicas, supone realmente un cambio de las estructuras de los centros escolares y una transformación del modelo curricular? La hipoteca de comprar libros de texto digitales, que ofrecen las poderosas editoriales, con dotaciones asociadas de tablets, le puede salir muy cara a una escuela que necesita renovarse metodológicamente. La pregunta que cabe realizarse es si con estas políticas de modernización conservadora de las instituciones escolares, colaboramos a perpetuar la fantasía de las expectativas en los dispositivos tecnológicos mientras neutralizamos iniciativas más centradas en otras metodologías como el aprendizaje por proyectos, que alejadas de los libros de texto, está más centrada en desarrollar un currículum renovado centrado en los intereses de los alumnos y profesores.

\footnotetext{
37 http://www.eskola20.euskadi.net/web/guest/familiak
} 


\subsection{Principado de Asturias}

La ausencia de inversiones para dotar a las escuelas de nuevos equipos y de mejores infraestructuras ha llevado a adoptar una política de amortización de los recursos proporcionados por la Consejería de Educación del Principado de Asturias, así como a apostar por el mantenimiento de los equipos y la explotación continuada de éstos. Si bien antes los alumnos de $5^{\circ}$ se llevaban los portátiles para utilizarlos hasta $2^{\circ}$ de la ESO, ahora estos ordenadores permanecen en el centro para ser utilizados por el resto de estudiantes de esos mismos niveles educativos.

Además, se han arbitrado fórmulas de innovación cualitativa para convertir las Tecnologías de la Información y Comunicación (TIC) en Tecnologías del Aprendizaje y Conocimiento (TAC), e impulsar prácticas didácticas concretas alejadas del mero manejo tecnológico. Concretamente, desde la Consejería de Educación se está impulsando la innovación en las escuelas a través de los denominados «Contratoprograma para la mejora del éxito escolar» junto a los «Proyectos de investigación e innovación educativa, dentro del marco del convenio de colaboración con la Universidad de Oviedo para realizar actividades educativas». Estos contratos-programa tienen como objetivo promover el éxito académico mediante el desarrollo de proyectos de Innovación Metodológica (AUlab). Con los que se pretende (BOPA, 2014):

- Promover modelos de gestión y organización ajustados a la realidad y necesidades de cada centro docente.

- Fomentar la coordinación y trabajo en equipo de los profesionales del centro implicados en el proceso de aprendizaje del alumnado.

- Mejorar la coordinación del centro docente con el entorno, optimizar el empleo de recursos y fomentar la participación real de las familias en la vida del centro.

- Impulsar la aplicación de cambios organizativos y metodológicos que permitan el seguimiento individualizado del alumnado para el desarrollo de sus competencias y dar respuesta a las necesidades educativas desde una perspectiva inclusiva, promoviendo la cultura de la autoevaluación como práctica cotidiana de los centros.

- Promover gradualmente la autonomía de los centros en la búsqueda de respuestas educativas que contribuyan al desarrollo de las competencias del alumnado.

El modelo de contrato-programa intenta que la innovación revierta en el éxito académico, atendiendo a las peculiaridades de cada centro y aula. Para ello, los centros disponen de cuatro años para implementar sus innovaciones metodológicas y valorar los resultados. Periodo durante el cual, la Administración se compromete a facilitarles los recursos materiales necesarios, el asesoramiento y apoyo externo de expertos, así como la formación específica que los docentes requieran para impulsar sus propias innovaciones, dentro del régimen de austeridad impuesto por la crisis económica. Puesto que se han reducido los programas de formación del profesorado con carácter presencial, reforzando los grupos de trabajo en centros, aprovechando para tal efecto las plataformas de teleformación, cursos online coordinados a través de los Centros de Profesores y Recursos (CPR).

También se han promovido convocatorias públicas para realizar proyectos bianuales de investigación e innovación educativa, mediante convenios de colaboración con la Universidad de Oviedo -sin dotación económica- para impulsar actividades educativas innnovadoras que fomenten la cooperación a través de proyectos interinstitucionales adscritos a cualquiera de las líneas prioritarias (Educastur, 2014): 
- Promover la convivencia y participación de todos los miembros de la Comunidad Educativa en las actividades del centro: profesorado, alumnado, familias y otros agentes sociales; y la adopción de estrategias específicas que favorezcan la convivencia y el respeto entre iguales.

- Potenciar el uso de estrategias metodológicas que promuevan el desarrollo de las competencias básicas y específicas del alumnado.

- Reconocer la diversidad funcional del alumnado y favorecer estrategias que refuercen el modelo de Educación Inclusiva.

- Fomentar la igualdad, promoviendo actividades para visibilizar y favorecer estrategias que refuercen el modelo de escuela coeducadora, tales como alentando la igualdad de representación del profesorado en los consejos escolares de los centros educativos, formándoles para ello.

- Implementar el uso adecuado de las Tecnologías de la información, del aprendizaje y de la comunicación (TIC/TAC). Conocer y explorar los usos didácticos que las TIC tienen para el aprendizaje y la enseñanza. Destacando que la finalidad de las Tecnologías del Aprendizaje y de la Comunicación (TAC), va más allá del mero aprendizaje de destrezas para el uso de TIC, apostando por la exploración de estas herramientas al servicio del aprendizaje y de la adquisición del conocimiento.

Otra iniciativa orientada a impulsar la innovación es la convocatoria de los Premios Impulso TIC Educación Asturias ${ }^{38}$, dirigidos al profesorado y alumnado de los centros educativos de Asturias, en las etapas de Infantil, Primaria, Secundaria, Bachillerato y Ciclos de Formación Profesional, organizados por el Colegio Oficial de Ingenieros en Informática del Principado de Asturias (COIIPA), el Colegio Oficial de Ingenieros Técnicos en Informática del Principado de Asturias (CITIPA) en colaboración con la Universidad Oviedo, a través de la Escuela Politécnica de Ingeniería de Gijón y la Escuela de Ingeniería Informática de Oviedo, con los que se pretende reconocer el trabajo realizado en los centros educativos y fomentar entre los estudiantes asturianos el interés por la informática, impulsando la creatividad, la capacidad analítica, la resolución de problemas, el trabajo en equipo y el auto-aprendizaje, sin duda son Premios que sirven de acicate en esta tarea.

Finalmente, hay que destacar que la fórmula de convenios con empresas es la vía que parece servir de balón de oxígeno para algunas escuelas, así la colaboración con Samsung a través del «Proyecto Samsung Smart School» está favoreciendo la integración de tabletas en algunos centros públicos. Esta empresa, en colaboración con el Ministerio de Educación, Cultura y Deporte, promueve la integración de las nuevas tecnologías en las aulas de un total de 52 centros educativos públicos pertenecientes a 14 Comunidades Autónomas, (junto con Ceuta y Melilla). En Asturias concretamente, se busca llegar a los centros escolares de zonas rurales, de forma especial en los cursos de quinto y sexto de Primaria. A los cuales se les ha proporcionado una tableta por alumno con aplicaciones educativas propuestas por el MEC, junto a recursos didácticos específicos recomendados por la Consejería y otros diseñados por la propia empresa tecnológica, una pantalla digital para el profesorado y un servidor que permita la conexión entre todos los dispositivos, encargándose además del seguimiento de la experiencia en cada centro, manteniendo una relación estrecha con los docentes, ofreciéndoles una formación básica y recabando sus opiniones y valoraciones sobre la puesta en práctica del proyecto.

\section{Conclusiones}

Para identificar las tendencias de las políticas educativas TIC que en estos momentos se están implementando en el contexto español, además de lo expuesto anteriormente con relación a lo que está ocurriendo en una muestra de Comunidades Autónomas, es necesario tener en cuenta también las

\footnotetext{
${ }^{38}$ http://www.impulsotic.org/semana/
} 
acciones que impulsa el Ministerio de Educación del Gobierno de España con relación a esta temática. Brevemente pudiéramos señalar que las mismas se caracterizan por.

- No existe una política o programa alternativo al Programa Escuela 2.0 destinado a impulsar de forma coordinada entre las Comunidades Autónomas los procesos de integración de las TIC en el sistema escolar español.

- Se ha reducido de forma muy notoria las subvenciones destinadas a los materiales didácticos tradicionales (como son los libros de texto) y prácticamente se han suprimido partidas económicas para la adquisición de recursos tecnológicos y de conectividad de los centros repercutiendo parte de los costes en las familias.

- Se apoya la creación y difusión de plataformas digitales de naturaleza privada destinadas a la oferta de contenidos educativos para ser empleados en el contexto escolar.

- Se consolida y potencia la incorporación al curriculum escolar tanto de Educación Primaria como de Educación Secundaria de la denominada «competencia digital» a trabajar en todos los cursos y materias.

A modo de conclusiones finales pudiéramos inferir que las tendencias de políticas educativas que se están dibujando actualmente en España sobre la integración de las TIC en las escuelas podríamos definirlas a partir de los siguientes rasgos ${ }^{39}$ :

a) Está emergiendo un discurso oficial (y oficioso) que propugna la lenta desaparición de las aulas de los libros de texto en papel y su sustitución por las plataformas de contenidos educativos digitales (Moral-Pérez \& Villalustre, 2014). Es el concepto de «mochila digital» empleado por los responsables políticos tanto en distintas Comunidades Autónomas como a nivel central del Ministerio de Educación. Los principales argumentos esgrimidos son tanto de naturaleza económica, en cuanto que se reducen los gastos de las administraciones públicas ya que el material didáctico impreso es más caro que el digital, como de naturaleza pedagógica ya que se afirma que estos contenidos favorecerán el desarrollo de la competencia digital y la adquisición de nuevos conocimientos en los estudiantes.

b) Otra tendencia es la de incorporar las tablets al aula en vez de los miniportátiles o PCs. Esto evidencia que las políticas educativas siguen teniendo como un eje central la dotación tecnológica de los últimos dispositivos consolidados en el mercado. A la vez, se están potenciando la tecnología inalámbrica para el acceso a la red en los centros y la creación de «espacios educativos con recursos» en la nube. Aunque no aparece siempre reflejado en la documentación de las políticas referidas comienza a aparecer el concepto de «mobile learning» (m-learning) como un modelo educativo a desarrollar desde las escuelas.

c) Se consolida la Pizarra Digital Interactiva (PDI) como dotación en todas las aulas de los centros educativos. Esta parece ser una tendencia común y generalizada en todas las Comunidades Autónomas siendo previsible que, a corto plazo, sea una realidad en gran parte de los colegios y aulas de forma que coexistan las pizarras tradicionales con las digitales. En este sentido la «conectividad» a la red también acompaña a las mismas por lo que la incorporación de las PDI como un elemento propio del aula del siglo XXI tiene que estar vinculado con la conexión a Internet.

d) En algunas Comunidades Autónomas se introduce el modelo BYOD (Bring Your Own Device) para el acceso a las tecnologías. Los profesores y estudiantes llevan al aula su propio dispositivo

\footnotetext{
39 Como podrá observarse algunas de estas tendencias van en la dirección de las apuntadas en el último informe del Horizon Report 2014 para la educación escolar aunque, lógicamente, no son necesariamente coincidentes (Johnson, Adams, Estrada \& Freeman, 2014).
} 
digital y con él acceden a los recursos didácticos. Se establecen diferentes escenarios de aplicación del modelo BYOD en un centro educativo: (a) dispositivo libre por parte de profesorado y estudiantes; (b) dispositivo seleccionado por profesorado y estudiantes de un listado elaborado por el centro educativo y (c) uso de un dispositivo específico, por parte de profesores y alumnos, aprobado por el centro educativo.

e) Como hemos señalado, las Comunidades Autónomas consolidan una tendencia desarrollada desde hace más de una década como es el impulsar los portales web o recursos educativos online propios de la Consejería de Educación dirigidos a su propio profesorado. Lo novedoso de estos portales es que no sólo ofrecen actividades o unidades didácticas digitales para la enseñanza de ciertas materias y asignaturas, sino que también ofrecen recursos online vinculados con la producción de información y comunicación por parte de profesores y estudiantes como son los blogs (tanto para el centro como las aulas), las wikis, y para favorecer la creación de redes sociales educativas.

f) También parece consolidarse la utilización del concepto de «aula virtual» vinculado con un LMS (en la mayor parte de los casos Moodle) ofertado para que los centros puedan crear sus espacios educativos. Por otra parte, una tendencia que parece confirmarse es el incremento de la oferta formativa institucional destinada al profesorado mediante la modalidad de «aulas virtuales».

\section{Referencias}

Alonso, C. (2012). La evolución de las políticas de uso de las TIC en la educación en Cataluña. En J.M. Sancho y C. Alonso (Comp.), La fugacidad de las políticas, la inercia de las prácticas: La educación y las tecnologías de la información y la comunicación (21-33). Barcelona: Octaedro.

Alonso, C.; Guitert, M. y Romeu, T. (2014). Los Entornos 1x1 en Cataluña. Entre las expectativas de las políticas educativas y las voces del profesorado. Educar, 50(1), 41-64.

Area, M. ; De Pablos, J.; Valverde, J. y Correa, J.M. (2010): Políticas educativas y buenas prácticas con TIC. Barcelona: Graó.

Area, M. (2006). Veinte años de políticas institucionales para incorporar las tecnologías de la información y comunicación al sistema escolar. En J.M Sancho (coord.). Tecnologías para transformar la educación. Madrid: AKAL/UIA.

Area, M. y Sanabria, A.L.. (2014): Opiniones, expectativas y valoraciones del profesorado participante en el Programa Escuela 2.0 en España. Educar, vol. 50/1, 15-39. Disponible en http://ddd.uab.cat/pub/educar/educar_a2014m16v50n1/educar_a2014m1-6v50n1p15.pdf_

Balanskat, A., Bannister, D., Hertz, B., Sigillò, E., \& Vuorikari, R. (2013). Overview and Analysis of 1:1 Learning Initiatives in Europe. JRC Scientific and Policy Report. Luxembourg: Publications Office of the European Union.

BOPA (2014). Resolución de 5 de mayo de 2014, de la Consejería de educación, Cultura y Deporte, por la que se aprueba la convocatoria del proceso de selección de centros docentes públicos dependientes de la Consejería de educación, Cultura y Deporte del Principado de Asturias para su incorporación al Programa "Contrato-Programa para la mejora del éxito escolar" en el curso 2014/2015. Boletín Oficial del Principado de Asturias, $n^{\circ} 106$ de 09-V-2014, 1-9. https: / sede.asturias.es/bopa/2014/05/09/2014-08094.pdf

De Pablos, J. (2012). Políticas educativas TIC en tiempos de crisis. El caso de Andalucía. Revista Campus Virtuales, n. 1, págs. 105 a 113. http:/ / www.revistacampusvirtuales.es/campusvirtuales/1/9.pdf

Del Moral, M.E. y Villalustre, L. (2014). Libros digitales: valoraciones del profesorado sobre el modelo de formación bimodal / Digital Books: teachers' assessment about the model of bimodal training. Revista Latinoamericana de Tecnología Educativa - RELATEC, 13(1), 89-99. $\quad$ Disponible en http://mascvuex.unex.es/revistas/index.php/relatec/article/view/1291

Fullan, M., Watson y Anderson, S. (2013): Ceibal: los próximos pasos. Informe final. Michael Fullan Enterprises, Toronto, Canadá. Disponible en http:/ /www.ceibal.org.uy/docs/FULLAN-Version-final-traduccion-Informe-Ceibal.pdf

Johnson, L., Adams Becker, S., Estrada, V., and Freeman, A. (2014): NMC Horizon Report: 2014 K-12 Edition . Austin, Texas: The New Media Consortium. Disponible en http:/ /cdn.nmc.org/media/2014-nmc-horizon-report-k12-EN.pdf

Losada, D.; Karrera, I. y Correa, J.M. (2011). Políticas sobre la integración de las TIC en la escuela de la Comunidad Autónoma del País Vasco. Revista Latinoamericana de Tecnología Educativa - RELATEC, 10(1), 21-35. Disponible en http://mascruex.unex.es/revistas/index.php/relatec/article/view/701 
Lugo, M.T. (2010). Las políticas TIC en la educación de América Latina.

Tendencias y experiencias. Revista Fuentes, 10, 52-68

OCDE (2010). 1:1 en Educación. Prácticas actuales, evidencias del estudio comparativo internacional e implicaciones en politicas. Madrid: Instituto de Tecnologías en http://www.ibertic.org/evaluacion/sites/default/files/biblioteca/28_1a1_en_educacion-ite_espana.pdf

One Laptop per Child (2011). One Laptop per Child deployment guide $2011 . \quad$ from http://wiki.laptop.org/images/1/1c/OLPC_Deployment_Guide_2011.pdf

Paredes Labra, J. (2012) Políticas educativas neoliberales para la integración de las TIC en educación. El caso de Madrid (España). Campus Virtuales. Revista científica iberoamericana de tecnología educativa. Vol 1, No 1, pág. 11-20 ISSN 22551514 Disponible on line: http://www.revistacampusvirtuales.es/index.php/revista/numeroactual/16-revista/21

Peirats, J. Sales, C. y San Martín, A. (2009): Un "portátil por estudiante" como argumento de disputa política en la sociedad digital. Educatio siglo XXI, 27, 2, págs. 53-69.

Pulido Bermejo, J.P. (2014). La experiencia de Extremadura: Portfolio de la Competencia Digital Docente. Presentado en IKANOS Workshop 2014. Recuperado a partir de http://ikanos.blog.euskadi.net/wpcontent/uploads/2014/05/20140512_CompetenciaDD_Extremadura_SSebastian_v2.pdf

Richardson, J., McLeod, S., Flora, K. and Sauers, N.J. (2013) Large-scale 1:1 computing initiatives: An open access database. International Journal of Education and Development using Information and Communication Technology. (IJEDICT), 2013, Vol. 9, Issue 1, pp. 4-18.

Sanabria, A.L. y Area, M. (2011) Las políticas educativas para incorporar las TIC al sistema escolar en Canarias. De Ábaco a ClicEscuela 2.0. En Roig Vila, R. \& Laneve, C. (Eds.) La práctica educativa en la sociedad de la información. Innovación a través de la investigación. La pratica educativa nella società dell'informazione. L'innovazione attraverso la ricerca Alcoy - Bresci: Marfil \& La Scuola Editrice pp. 369-381.

Sola, M. (2012). 10 años de TIC. Andalucía Educativa. Revista Digital. Disponible en http://www.juntadeandalucia.es/educacion/webportal/web/revista-andalucia-educativa/enportada/-/noticia/detalle/10-anos-de-tic-miguel-sola-fernandez-universidad-de-malaga-1

VV.AA. (2012). Conclusiones finales de las Jornadas. Las TIC en la educación de Andalucía: presente y futuro. Consejería de Educación, Cultura y Deporte. Junta de Andalucía. Disponible en http://www.juntadeandalucia.es/educacion/portal/com/bin/Contenidos/IEFP/INNOVACION/20130111_TIC/13 57893644151_documento2_conclusiones_finales_jornadastic.pdf

Valverde-Berrocoso, J. (Coord.) (2014). Políticas educativas para la integración de las TIC en el sistema educativo. El caso de Extremadura, Madrid: Dykinson. 
